\title{
Microphone Array and Raspberry Pi Interfacing for Real-time DOA Estimation and Tracking of Audio Sources
}

\author{
Ashish Patwari ${ }^{*}$, Saicharan $M^{1}$, Sricharan $L^{1}$, Hemanth Penta ${ }^{1}$ \\ ${ }^{1}$ School of Electronics Engineering, Vellore Institute of Technology, Vellore, Tamil Nadu, India. \\ *ashish.p@ vit.ac.in
}

\begin{abstract}
Microphone arrays are commonly used for Direction of Arrival (DOA) estimation of audio sources. This paper presents the design and implementation of a real-time embedded system (RTES) for audio source localization. A commercially available microphone array assembly (consisting of four microphones) has been interfaced to Raspberry $\mathrm{Pi} 3 \mathrm{~B}$ for estimating the arrival angles of audio/sound/acoustic sources. The acoustic signals received a teach of the microphones are used for DOA estimation using the Generalized Cross Correlation Phase Transform (GCC-PHAT) algorithm. The estimated DOA is then displayed on the serial monitor. Servo motors are interfaced to the Raspberry Pi and are programmed to point towards the estimated direction of the audio source (on the basis of the estimated DOA value). The estimated DOA value is also updated to the cloud-based server Firebase using the inbuilt Wi-Fi on Raspberry Pi, and can be remotely accessed through a smartphone running on android platform. The proposed system has been designed and tested successfully and it is observed that it provides accurate DOA estimation and audio source tracking in real-time. This has various uses in applications like voice assistant robots, machine audition, hearing aids, source remixing, augmented reality headsets, security cameras, sound tracking (sonar sensor) devices, bird identification, mammal localization and so on.
\end{abstract}

Key words: Array Processing, Direction of Arrival Estimation, Microphone arrays, Raspberry Pi, Sound Source Localization.

\section{INTRODUCTION}

Array signal processing is a scientific field of study which involves the processing of information-bearing signals that are received by an array of sensors operating in an environment of interest [1]. The environment may be on the ground, above the ground, under the water or in outer space. An array contains two or more sensors whose outputs are combined constructively. The elements are arranged in a specific geometrical layout. An array has better directional properties than an individual sensor. Arrays can have different geometries - such as linear, planar, circular, hexagonal, spherical etc. It is easy to predict that an array spanning across more dimensions can extract more details about the scene of interest.

Sensor arrays find application in diverse fields such as Radio Detection and Ranging (RADAR), Space exploration, Sound Navigation and Ranging (SONAR), chemical sensing, Seismology, medical imaging (ultrasound), wireless communications, navigation, source localization, optical communication, fault location in rotating machinery (fan noise, wind turbine noise, rotating blade noise) etc. Depending on the application, the sensors could be antennas, microphones, hydrophones, geophones, ultrasonic probes etc[1], [2]. For example, hydrophone arrays are used in sonar, acoustic arrays are used for audio source localization, piezoelectric sensors are used in medical ultrasound, geophone arrays are used in seismology etc. More specifically, antenna arrays are used for electromagnetic applications such as radar, radio astronomy, remote sensing, wireless communications, positioning and navigation[1], [3]-[9].

In many of the above applications, it is of primary interest to sense the angles from which the source signals arrive at the receiver. The signal of interest may be an electromagnetic wave, a sound wave, an underwater acoustic wave, etc[10]. A sensor array can determine the directions/angles from which the signals originate. Individual sensors cannot sense direction. Unlike individual sensors, a sensor array has a sense of direction and can estimate the direction of arrival (DOA) of incoming signals based on the relative phase difference between the signals received at its elements. Therefore, sensor arrays are widely used for DOA estimation in various fields [11], [12].

Microphone arrays are commonly used in a variety of audio and speech processing systems such as robot audition, machine listening, hearing aids, wild life localization and tracking, speech enhancement, sound recording, ambient assisted living etc[13], [14].

Different techniques such as Time of Arrival (TOA) estimation, Time difference of Arrival (TDOA) method, DOA estimation and Steered response power (SRP) beamforming methods are available for audio localization. A detailed comparison of these algorithm families for audio source localization in wireless acoustic sensor networks has been excellently reviewed in [15]. The use of microphone arrays in present-day sound recording and spatial audio techniques was dealt at great lengths in [16]. Sparse arrays are known to offer higher degrees of freedom than regular arrays for the same number of sensors. Alternately, they need fewer sensors to offer the same aperture as regular/filled arrays [17]-[19]. Of 
late, sparse microphone arrays have made their way into audio source localization in recent times [20], [21].

The rest of the paper is organized as follows. Section 2 describes the existing methods. Section 3 gives the proposed method. Section 4 presents the methodology followed for designing the prototype and experimental setup. Section 5 presents the results. Section 6 concludes the paper.

\section{EXISTING METHODS}

Audio source localization has been widely studied in the past. However, most of the studies are theoretical and simulation-based as they involve the development of signal processing techniques for overcoming sensor noise, mutual coupling, obtaining accurate DOA estimation in the presence of fewer snapshots, operation in harsh and low signal-to-noise-ratio (SNR) conditions etc[22]-[24]. Another portion of research papers is devoted to the hardware design of microphone arrays[25]-[28].An outdoor sound source localization system wherein a microphone array was fitted to a drone was demonstrated in [29].A huge microphone array with 512 microphones was designed and demonstrated in [30]. Similarly, a 1020-node microphone array was designed and tested successfully [31].

Field programmable gate arrays (FPGAs) and digital signal processors (DSP) have been widely demonstrated in the literature for acoustic source localization[32]. A detailed review on the use of FPGA platforms for acoustic beamforming using microphone arrays has been presented in [33]. The paper highlighted current trends, difficulties and future opportunities in the given area. One of the early FPGA implementations for real-time sound localization was presented in [34].A system for blind source separation from a mixture of multi-channel data has been implemented in [35].

Based on the existing literature, we have conceptualized that there is a need for plug-and-play interface for DOA estimation. Hence, we have selected a commercially available microphone array, namely, the Respeaker 4-mic array which has inbuilt facilities for multi-channel sound acquisition, data conversion and an inherent support for DOA estimation. The Respeaker array is optimized for interfacing with the Raspberry Pi and the computational power of Raspberry Pi neatly complements the real-time DOA estimation requirement using the signals acquired at multiple channels of the Respeaker array.

\section{PROPOSED METHOD}

In this section, we present the proposed method. Two main hardware components used in this work are Raspberry Pi and the Respeaker microphone array. A system which is very similar in concept and design has been presented in [36], but needs an FPGA board and a Raspberry Pi. In contrast, the system proposed in the present work does not need any FPGA support. To the best of our knowledge, this is the first time that a real-time audio source location and tracking mechanism is proposed solely on the basis of a microphone array and Raspberry Pi.

\subsection{System Operation}

A high-level schematic of the proposed system operation is shown in Figure 1. It can be seen that the Respeaker array captures the audio signals from the external environment, converts them into digital form and sends the data to Raspberry Pi for DOA estimation. The DOA algorithm is executed inside the Raspberry $\mathrm{Pi}$ and the estimated angle is displayed on to a serial monitor. Simultaneously, a command is given to the servo motors to rotate and point towards the estimated angle of arrival. The estimated DOAs are also updated on the IoT cloud using the Firebase platform and can be remotely accessed using an android smartphone for Internet of Things (IoT) applications.

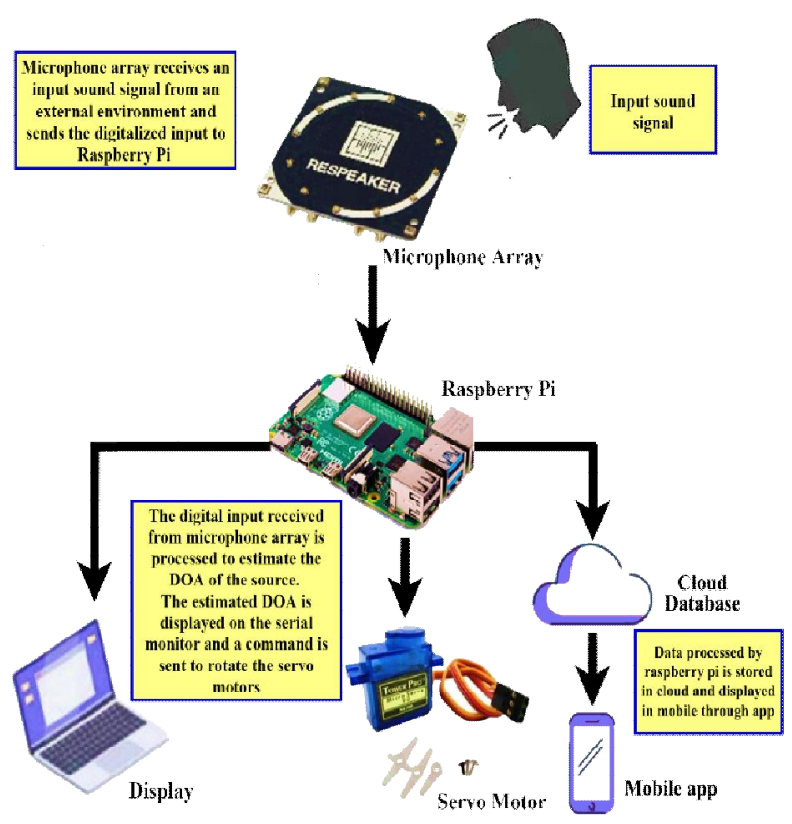

Figure 1:Outline of the proposed system operation.

\subsection{Hardware Description}

Figure 2 shows the hardware block diagram of the proposed system.

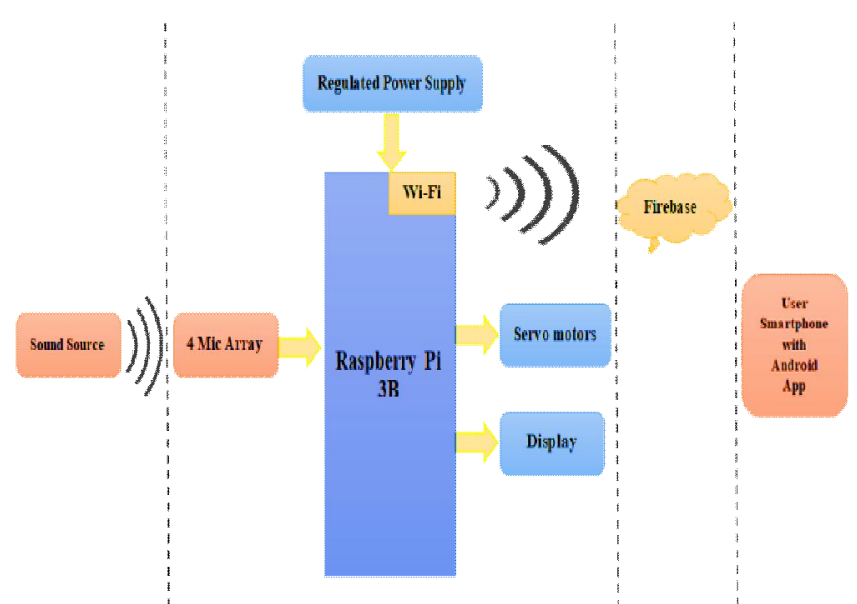

Figure 2: Hardware block diagram of the proposed system

It can be seen that the inbuilt Wi-Fi feature available in Raspberry Pi 3B model has been used to send the DOA 
estimation data to the Firebase cloud. The user can then access the data on a continuous basis using his/her smartphone by using the appropriate android app.

\section{METHODOLOGY}

The methodology followed for the hardware design and testing of the proposed audio source localization system is presented below.

\subsection{Software Flowchart}

The flow chart for the operation of the proposed system is shown in Figure 3. First, the Voice Activity Detection (VAD) module has to be enabled. It is seen that the microphones capture the sound only when the sound intensity exceeds the preset threshold. The quad channel data acquired by the Respeaker is digitized and sent to Raspberry Pi for further processing.

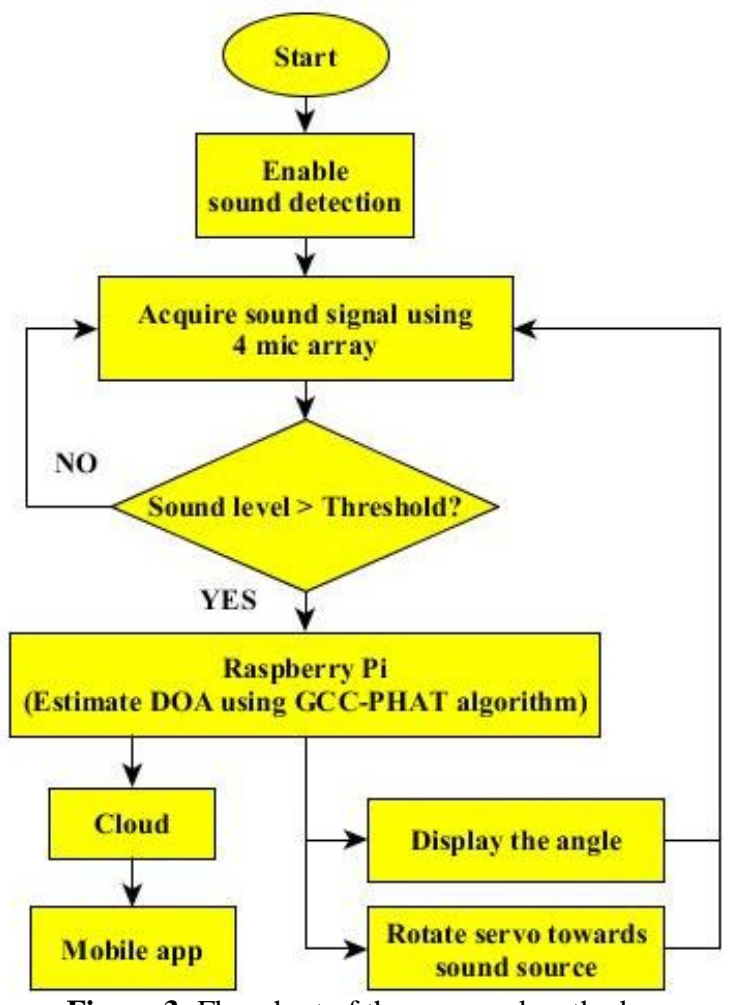

Figure 3: Flowchart of the proposed method

\subsection{Algorithm Details}

The Respeaker has inbuilt support for DOA estimation using the Generalized cross correlation-phase transformation (GCC-PHAT). It is well-known that subspace-based DOA estimation algorithms such as the MUSIC, ESPRIT and Root MUSIC cannot work in the presence of coherent arrivals. Two signals are coherent if one is a scaled and shifted version of the other. Multipath effect and deliberate jamming are the main causes for coherent arrivals[37]. Pair-wise coherent sources occur in a two-ray model or in a situation where there is exactly one jammer per source signal. Multipath can occur in undersea acoustic channels (sonar), aero-acoustic channels (due to reverberation), radars, and wireless communications. Two sources become coherent when they are fully correlated.
Hence, an additional intermediate step of spatial smoothing is needed to equip these algorithms to detect coherent sources. Spatial smoothing is a pre-processing technique to restore the rank of the source covariance matrix [38]-[40]. While subspace methods can estimate the angles of multiple sources at once, the GCC-PHAT is applicable only when there is a single sound source.

Nevertheless, GCC-PHAT has been widely recognized to operate accurately in the presence of reverberating acoustic channels[41], [42]. Hence, we consider only GCC-PHAT in this paper. Figure 4 explains the main steps followed in the GCC-PHAT algorithm.

GCC-PHAT is based on the time difference of arrival (TDOA) scheme. In this, the sensor array is assumed to be in the far field of the sound source such that the source is perceived at the same angle by all the sensors in the sensor array. Pair-wise cross-correlation between the signals received at each sensor pair yields the time delay between signal arrivals at each sensor. Once all the time delays between every sensor pair are obtained, a least squares approximation is made use of to obtain the DOA of the source.

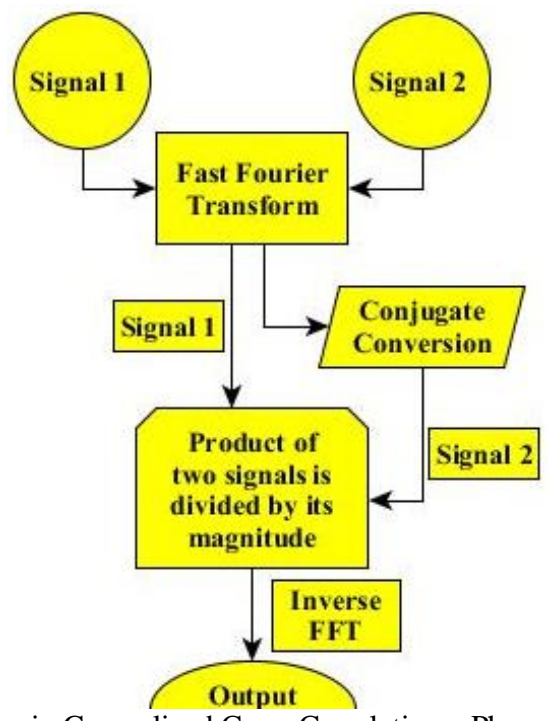

Figure 4: Steps in Generalized Cross Correlation - Phase Transform (GCC-PHAT)

\subsection{Hardware Interfacing}

The pin-level connections for interfacing the Respeaker array with the Raspberry Pi are given in Table 1.

Table 1:Usage of Raspberry Pi pins

\begin{tabular}{|l|l|}
\hline Pin Number & Pin Function \\
\hline Pin no. 32 & $\begin{array}{l}\text { GPIO - 12 [To drive } \\
\text { Servo Motor 1] }\end{array}$ \\
\hline Pin no. 33 & $\begin{array}{l}\text { GPIO - 13 [To drive } \\
\text { Servo Motor 2] }\end{array}$ \\
\hline Pin no. 4 & VCC \\
\hline Pin no. 6 & Ground \\
\hline Other 36 pins & Mic array \\
\hline
\end{tabular}




\section{EXPERIMENTAL RESULTS}

A working prototype of the proposed system was built and tested for functionality. The system works as per the initial design goals. Figures 5 and 6 show the hardware prototype.

When a person is speaking around the microphone array, the microphones pick up the sound signal and send it to the onboard Analog to Digital Converter (ADC) which is available on Respeaker. After A/D conversion, the digital data from four channels of the Respeaker is sent to Raspberry Pi using the I2S pins. The data obtained from the microphone array is then processed in the Raspberry $\mathrm{Pi}$ using the GCC-PHAT algorithm as mentioned earlier. Once, the Raspberry Pi estimates the DOA of the sound source, the value is displayed onto the serial terminal and also fed to the servo motor so that it can rotate towards the estimated angle.

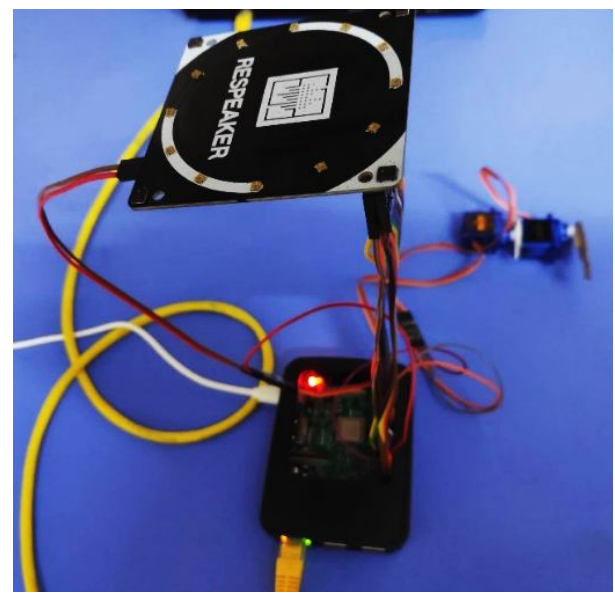

Figure 5: Hardware Prototype

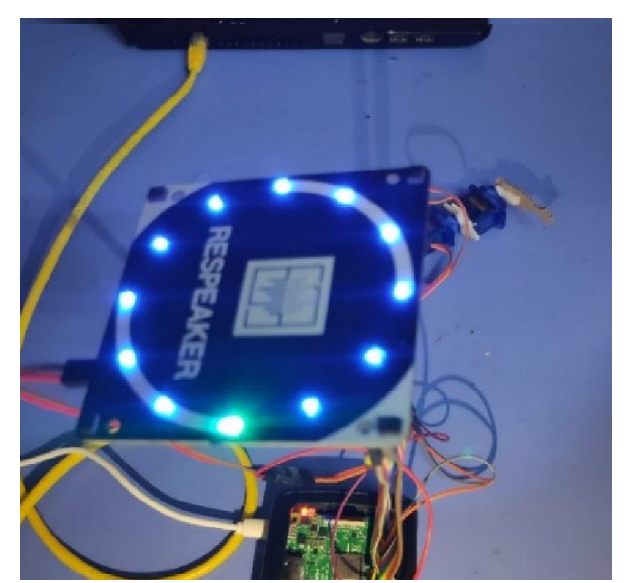

Figure 6:The LED ring on the Respeaker glows when voice activity is detected

5.1 DOA estimation procedure using the proposed system For DOA estimation, the user has to utter the keyword Snowboy within the vicinity of the Respeaker. The Respeaker can capture sound within a range of $3 \mathrm{~m}$. Figure 7 shows one of the authors speaking the keyword. Figure 8 is when Voice Activity Detection (VAD) is sensed by the device.

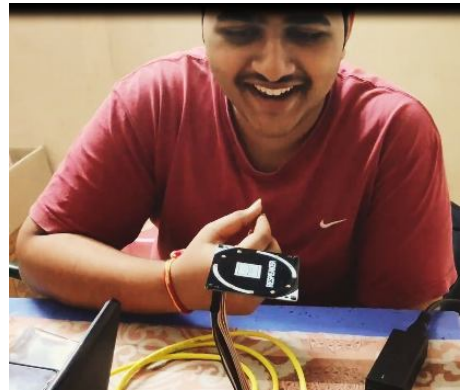

Figure 7: DOA estimation trial - User speaks the keyword Snowboy

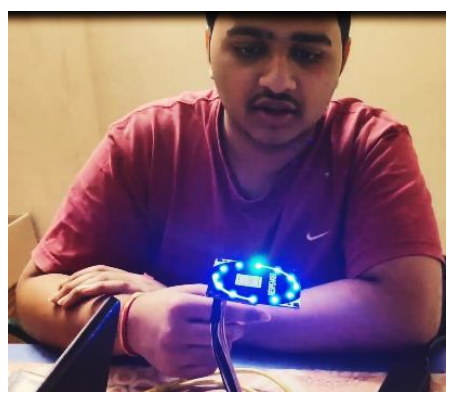

Figure 8: DOA estimation trial - Device detects voice activity

After this, the sound signals captured by the four microphones of the Respeaker are passed through A/D converters and given as an input to the Raspberry Pi which determines the DOA of the source using the inbuilt GCC-PHAT algorithm. The estimated angle is then displayed on the screen as shown in Figure 9. It can be seen that the Respeaker perceives the speaker at an angle of $82^{\circ}$.

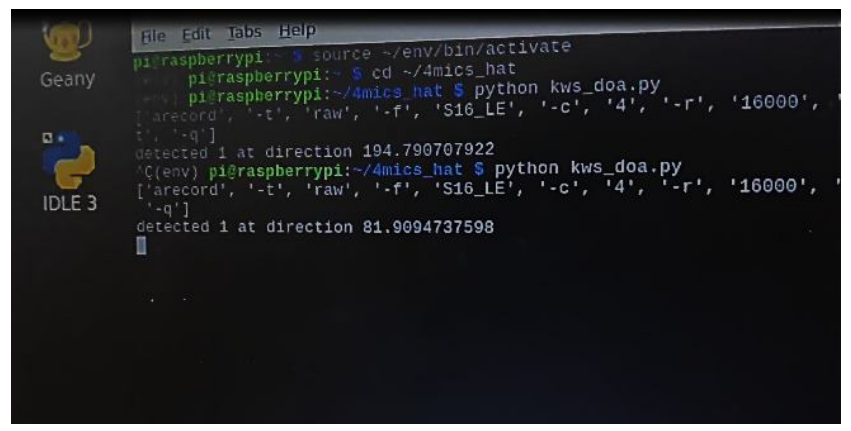

Figure 9:Estimated angle is displayed on the screen

After obtaining these basic results, we have programmed the setup in such a way that the DOA angle is finalized after waiting for five detection cycles. This helps to stabilize the location of the speaker.

\subsection{Sound Source at $151^{\circ}$}

Next, we demonstrate the use of servo motors and show how the DOA estimation results obtained at the Respeaker can be remotely accessed using an android smartphone. Consider that the speaker is at an angle of $151^{\circ}$ relative to the microphone array. By following the procedure given in subsection 5.1, the DOA estimation is carried out and the estimated angle is displayed on to the screen as shown in Figure 10. 
The estimated angle is fed as an input to the servo motor assembly. Figure 11 shows that the servo motor rotates and points to the desired angle. Next, the information about the estimated angle is transferred to the cloud using Firebase. The user can then access the information using his/her smartphone as shown in Figure 12.

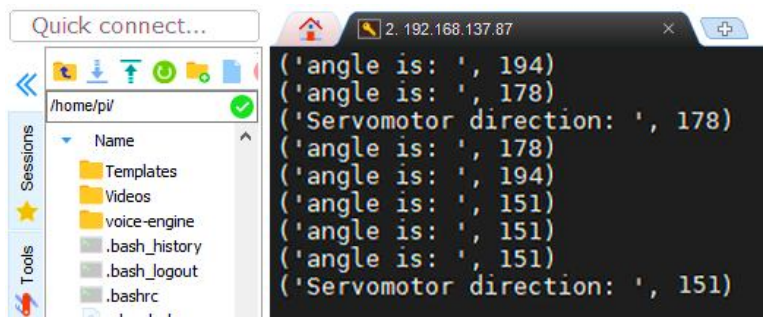

Figure 10: DOA detected at $151^{\circ}$

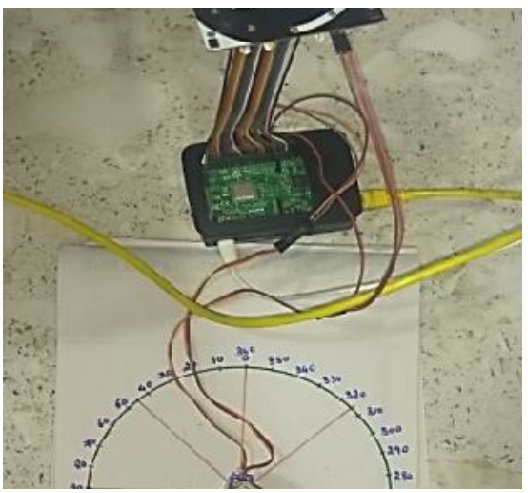

Figure 11: Servo motor pointing towards the estimated angle

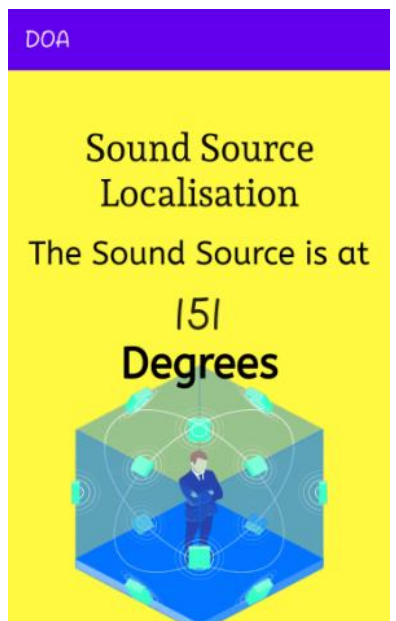

Figure 12: Android application in smartphone which provides remote updates related to the estimated angles

\subsection{Sound Source at $45^{\circ}$}

Similar results have been obtained when the speaker was positioned at $45^{\circ}$ relative to the Respeaker and are shown in Figures 13 and 14.

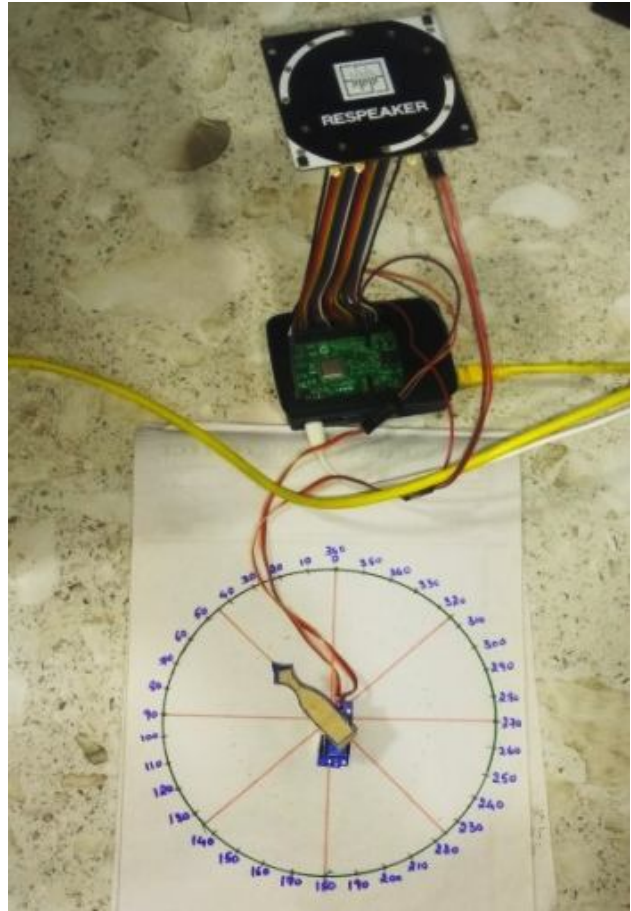

Figure 13: Servo points towards $45^{\circ}$, the estimated DOA

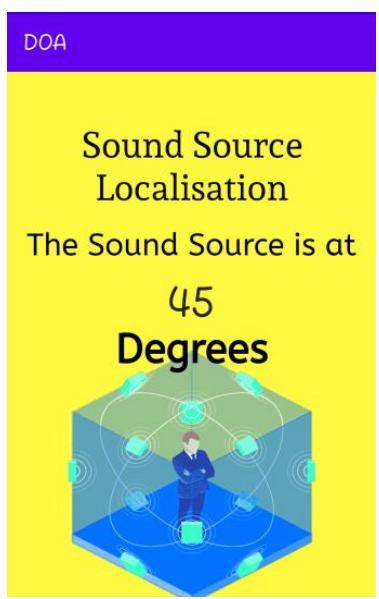

Figure 14: App data when the corresponding to the source at $45^{\circ}$

Hence, all the features of the proposed system have been designed and demonstrated successfully.

\section{CONCLUSION AND FUTURE SCOPE}

A real-time embedded system for estimating the DOAs of sound sources using microphone arrays is demonstrated here. Servo motors are used to point towards the estimated directions from which the sound signal is perceived by the microphone array.

As a future enhancement, a camera module can be added to this device to record and transmit the video feed. The camera has to be placed on the servo motor platform so that it can rotate towards the direction of the sound source. This will be useful for surveillance applications as well as for speaker tracking in meeting rooms. The video footage being recorded at the site can be viewed remotely using Team viewer software. Additionally, the Respeaker can be used to perform 
adaptive receive beamforming in order to spatially tune into a particular speaker's voice in meeting rooms by suitable beam steering and null placement.

\section{REFERENCES}

[1] S. Haykin, J. P. Reilly, V. Kezys, and E. Vertatschitsch, Some aspects of array signal processing, IEE Proc. $F$ Radar Signal Process., vol. 139, no. 1, pp. 1-26, Feb. 1992, doi: 10.1049/ip-f-2.1992.0001.

[2] S. Theodoridis and R. Chellappa, Academic Press Library in Signal Processing, Volume 3: Array and Statistical Signal Processing, 1st ed. Academic Press, 2013.

[3] A. Patwari and G. R. Reddy, $1 D$ direction of arrival estimation using root-MUSIC and ESPRIT for dense uniform linear arrays, in 2017 2nd IEEE International Conference on Recent Trends in Electronics, Information Communication Technology (RTEICT), May 2017, pp. 667-672, doi: 10.1109/RTEICT.2017.8256681.

[4] S. Kutty and D. Sen, Beamforming for Millimeter Wave Communications: An Inclusive Survey, IEEE Commun. Surv. Tutor., vol. 18, no. 2, pp. 949-973, Secondquarter 2016, doi: 10.1109/COMST.2015.2504600.

[5] M. Wang, F. Gao, S. Jin, and H. Lin, An Overview of Enhanced Massive MIMO With Array Signal Processing Techniques, IEEE J. Sel. Top. Signal Process., vol. 13, no. 5, pp. 886-901, Sep. 2019, doi: 10.1109/JSTSP.2019.2934931.

[6] W. Hong, K. H. Baek, and S. Ko, Millimeter-Wave 5G Antennas for Smartphones: Overview and Experimental Demonstration, IEEE Trans. Antennas Propag., vol. 65, no. 12, pp. 6250-6261, Dec. 2017, doi: 10.1109/TAP.2017.2740963.

[7] R. A. Monzingo, R. Haupt, and T. Miller, Introduction to Adaptive Arrays, 2nd Edn. Institution of Engineering and Technology, 2011.

[8] L. C. Godara, Smart Antennas, CRC Press, Jan. 27, 2004.

https://www.crcpress.com/Smart-Antennas/Godara/978 0849312069 (accessed Mar. 31, 2016).

[9] A. E. Fadl, Estimation Direction Arrival of Smart Antenna, Int. J. Emerg. Trends Eng. Res., vol. 8, no. 4, pp. 1051-1055, 2020, doi: 10.30534/ijeter/2020/16842020.

[10] P. S. Naidu, Sensor Array Signal Processing, Second Edition. CRC Press, 2009.

[11] C. A. Balanis, Antenna Theory: Analysis and Design, Third edition. Wiley, 2009.

[12] S. Bellofiore, C. A. Balanis, J. Foutz, and A. S. Spanias, Smart-antenna systems for mobile communication networks. Part 1. Overview and antenna design, IEEE Antennas Propag. Mag., vol. 44, no. 3, pp. 145-154, Jun. 2002, doi: 10.1109/MAP.2002.1039395.

[13] B. Widrow and F.-L. Luo, Microphone arrays for hearing aids: An overview, Speech Commun., vol. 39, no. 1, pp. 139-146, Jan. 2003, doi: 10.1016/S0167-6393(02)00063-8.
[14] M. Brandstein and D. Ward, Eds., Microphone Arrays: Signal Processing Techniques and Applications. Berlin Heidelberg: Springer-Verlag, 2001.

[15] M. Cobos, F. Antonacci, A. Alexandridis, A. Mouchtaris, and B. Lee, A Survey of Sound Source Localization Methods in Wireless Acoustic Sensor Networks, Wireless Communications and Mobile Computing, 2017. https://www.hindawi.com/journals/wcmc/2017/3956282 / (accessed May 20, 2020).

[16] J. Y. Hong, J. He, B. Lam, R. Gupta, and W.-S. Gan, Spatial Audio for Soundscape Design: Recording and Reproduction, Appl. Sci., vol. 7, no. 6, p. 627, Jun. 2017, doi: 10.3390/app7060627.

[17] A. Patwari and G. R. Reddy, A Conceptual Framework for the Use of Minimum Redundancy Linear Arrays and Flexible Arrays in Future Smartphones, Int. J. Antennas Propag., vol. 2018, no. 9629837, p. 12, 2018, doi: $10.1155 / 2018 / 9629837$.

[18] C.-L. Liu and P. P. Vaidyanathan, Cramér-Rao bounds for coprime and other sparse arrays, which find more sources than sensors, Digit. Signal Process., vol. 61, pp. 43-61, Feb. 2017, doi: 10.1016/j.dsp.2016.04.011.

[19] M. Yang, A. M. Haimovich, X. Yuan, L. Sun, and B. Chen, A Unified Array Geometry Composed of Multiple Identical Subarrays With Hole-Free Difference Coarrays for Underdetermined DOA Estimation, IEEE Access, vol. 6, pp. 14238-14254, 2018, doi: 10.1109/ACCESS.2018.2813313.

[20] J. Zhao and C. Ritz, Co-prime Circular Microphone Arrays and Their Application to Direction of Arrival Estimation of Speech Sources, in ICASSP 2019 - 2019 IEEE International Conference on Acoustics, Speech and Signal Processing (ICASSP), May 2019, pp. 800-804, doi: 10.1109/ICASSP.2019.8682876.

[21] F. S. Rawnaque and J. R. Buck, Comparing the effect of aperture extension on the peak sidelobe level of sparse arrays, J. Acoust. Soc. Am., vol. 142, no. 5, pp. EL467-EL472, Nov. 2017, doi: 10.1121/1.5009112.

[22] M. Debrouwere and D. Angland, Airy pattern approximation of a phased microphone array response to a rotating point source, J. Acoust. Soc. Am., vol. 141, no. 2, pp. 1009-1018, Feb. 2017, doi: 10.1121/1.4976068.

[23] B. Tóth, J. Vad, and G. Kotán, Comparison of the Rotating Source Identifier and the Virtual Rotating Array Method, Period. Polytech. Mech. Eng., vol. 62, no. 4, pp. 261-268, Jul. 2018, doi: 10.3311/PPme.11194.

[24] R. Rabenstein and P. Annibale, Acoustic Source Localization under Variable Speed of Sound Conditions, Wireless Communications and Mobile Computing,

2017. https://www.hindawi.com/journals/wcmc/2017/9524943 / (accessed May 20, 2020).

[25] X. Zhang, Z. Chu, Y. Yang, S. Zhao, and Y. Yang, An Alternative Hybrid Time-Frequency Domain Approach Based on Fast Iterative Shrinkage-Thresholding Algorithm for Rotating Acoustic Source Identification, IEEE Access, vol. 7, pp. 59797-59805, 2019, doi: 10.1109/ACCESS.2019.2915001. 
[26] G. Chen and Y. Xu, A Sound Source Localization Device Based on Rectangular Pyramid Structure for Mobile Robot, J. Sens., vol. 2019, p. e4639850, 2019, doi: $10.1155 / 2019 / 4639850$.

[27] H. Xing and X. Yang, Sound Source Localization Fusion Algorithm and Performance Analysis of a Three-Plane Five-Element Microphone Array, Appl. Sci., vol. 9, no. 12, p. 2417, Jan. 2019, doi: 10.3390/app9122417.

[28] S. Jekosch and E. Sarradj, An Extension of the Virtual Rotating Array Method Using Arbitrary Microphone Configurations for the Localization of Rotating Sound Sources, Acoustics, vol. 2, no. 2, pp. 330-342, Jun. 2020, doi: 10.3390/acoustics2020019.

[29] K. Hoshiba et al., Design of UAV-Embedded Microphone Array System for Sound Source Localization in Outdoor Environments, Sensors, vol. 17, no. 11, p. 2535, Nov. 2017, doi: 10.3390/s17112535.

[30] H. F. Silverman, W. R. Patterson, and J. L. Flanagan, The huge microphone array, IEEE Concurr., vol. 6, no. 4, pp. 36-46, Oct. 1998, doi: 10.1109/4434.736423.

[31] E. Weinstein, K. Steele, A. Agarwal, and J. Glass, LOUD: A 1020 node Microphone Array and Acoustic Beamformer, Courant Institute of Mathematical Sciences New York United States, Jul. 2007. Accessed: May 20, 2020. [Online]. Available: https://apps.dtic.mil/seo/citations/AD1020161.

[32] M. D. K. Das and M. Priyanka, FPGA implementation of Acoustic Source Localization algorithm, in 2016 International Conference on Wireless Communications, Signal Processing and Networking (WiSPNET), Mar. 2016, pp. 146-150, doi: 10.1109/WiSPNET.2016.7566109.

[33] B. Da Silva, A. Braeken, and A. Touhafi, FPGA-Based Architectures for Acoustic Beamforming with Microphone Arrays: Trends, Challenges and Research Opportunities, Computers, vol. 7, no. 3, p. 41, Sep. 2018, doi: 10.3390/computers7030041.

[34] D. Nguyen, P. Aarabi, and A. Sheikholeslami, Real-time sound localization using field-programmable gate arrays, in 2003 IEEE International Conference on Acoustics, Speech, and Signal Processing, 2003. Proceedings. (ICASSP '03)., Apr. 2003, vol. 2, pp. II-573, doi: 10.1109/ICASSP.2003.1202431.

[35] M. Mekhfioui, A Comparative Approach of Blind Source Separation with Arduino Due and TMS320C6713, Int. J. Emerg. Trends Eng. Res., vol. 8, no. 3, pp. 864-867, Mar. 2020, doi: 10.30534/ijeter/2020/42832020.

[36] Sound Source Localization | Innovation in Augmented Listening Technology - University of Illinois at Urbana-Champaign." Blog post https://publish.illinois.edu/augmentedlistening/sound-so urce-localization/ (accessed May 20, 2020).

[37] H. Krim and M. Viberg, Two decades of array signal processing research: the parametric approach, IEEE Signal Process. Mag., vol. 13, no. 4, pp. 67-94, Jul. 1996, doi: 10.1109/79.526899.

[38] J. E. Evans, J. R. Johnson, and D. F. Sun, Application of Advanced Signal Processing Techniques to Angle of
Arrival Estimation in ATC Navigation and Surveillance Systems, Lincoln Laboratory, Technical Report, Jun. 1982. Accessed: Apr. 30, 2018. [Online]. Available: http://dspace.mit.edu/handle/1721.1/97639.

[39] T.-J. Shan, M. Wax, and T. Kailath, On spatial smoothing for direction-of-arrival estimation of coherent signals,IEEE Trans. Acoust. Speech Signal Process., vol. 33, no. 4, pp. 806-811, Aug. 1985, doi: 10.1109/TASSP.1985.1164649.

[40] S. U. Pillai and B. H. Kwon, Forward/backward spatial smoothing techniques for coherent signal identification, IEEE Trans. Acoust. Speech Signal Process., vol. 37, no. 1, pp. 8-15, Jan. 1989, doi: 10.1109/29.17496.

[41] Y. (Arden) Huang, J. Benesty, and J. Chen, Time Delay Estimation and Source Localization, in Springer Handbook of Speech Processing, J. Benesty, M. M. Sondhi, and Y. A. Huang, Eds. Berlin, Heidelberg: Springer, 2008, pp. 1043-1063.

[42] L. Chen, Y. Liu, F. Kong, and N. He, Acoustic Source Localization Based on Generalized Cross-correlation Time-delay Estimation, Procedia Eng., vol. 15, pp. 4912-4919, Jan. 2011, doi: 10.1016/j.proeng.2011.08.915. 